\title{
Motion of nanoscale contaminant particles in air bearings under electrostatic charges: a case study
}

\author{
B. W. Yeigh ${ }^{1}$, R. H. Polwort ${ }^{2}$ \& G. S. Gipson ${ }^{3}$ \\ ${ }^{I}$ Office of the President, \\ State University of New York Institute of Technology, USA \\ ${ }^{2}$ Plant Engineering, The Charles Machine Works, Inc., USA \\ ${ }^{3}$ Department of Civil \& Environmental Engineering, \\ Oklahoma State University, USA
}

\begin{abstract}
This case study examines the possible effect that electrostatic forces may have on debris particle motion through the interface between two sliding surfaces in an air bearing. It uses as an example the read/write head and disk surface of a typical modern hard disk drive. The Reynolds equation for compressible fluids at high bearing numbers is solved using a factored implicit scheme. A first-order molecular slip correction is also applied. Debris particle motion equations are derived with corrections for gravity, drag, and the Saffman lift effect from previous studies and combined with an equation for electrostatic forces proposed in this study. Aluminum debris particles ranging from 150 to $300 \mathrm{~nm}$ are introduced and tracked by solving motion equations using Runge-Kutta methods. Electrostatic forces acting on a debris particle were found to affect trajectories at levels of charge far lower than anticipated. Charges at levels as low as 1.5 electrons influenced particle motion significantly.

Keywords: molecular slip, Reynolds equation for compressible fluids, nano particles, factored implicit scheme, Runge-Kutta, surface mechanics, electrostatic forces, particle contamination.
\end{abstract}

\section{Introduction}

Air has a number of advantages as a lubricant: abundant supply, cleanliness, and lack of environmental and health issues associated with its use compared to a 
petroleum-based product. However, because of its low viscosity, the speed of an air-lubricated bearing must be several times higher than an oil-lubricated bearing to support the same load. Even though the high speeds required by the air bearing preclude its use in some heavy load applications, there are just as many applications where air bearings are suited or even more ideal than oil-lubricated bearings. They include: machine tool spindles, turbo-machinery, instrument bearings such as gyroscopes, dental drills, textile processing devices, and magnetic media data recording devices (disk drives).

Loose particle(s) inside disk drives (e.g., air bearings) can be detrimental. With air bearing clearance in the sub 30nm level, the particle size does not have to be large to cause serious damages to the disk. Failed disk drives show buildup of debris on the head, possibly from loose particles and lubricant. Whether the loose particles come from fine particles accumulated on the leading edge tapers [10] or from contaminant whiskers that broke off from the trailing edge [8], these particles can lead to third-body abrasions of disk surface. Several questions arise. First, inside the air bearing, where do trapped particles go? Do they adhere to the slider/disk or wash out of the bearing? What operating conditions force loose particles to move towards the slider? When do they move toward the disk surface?

\section{Numerical solution for the compressible Reynolds equation}

Relying on the pressure generated by the relative motion of sliding surfaces, geometry, and fluid viscosity, hydrodynamic bearings push the contact surfaces apart. Through the converging gap, the fluid enters through the higher of the two opening known as the leading edge and exits the lower trailing edge by the relative motion of the surfaces. The Reynolds equation calculates the pressure generated between the two surfaces. In the continuum form, the differential equation is obtained from the Navier-Stokes and continuity equations. Derivations are easily found $[2,7,16]$.

In the derivation, surfaces are assumed smooth and contain negligible traction. The Newtonian fluid between the surfaces obeys laminar flow rules. Constant fluid viscosity and isothermal conditions also apply. The inertial forces within the fluid are neglected. At the boundaries, nonslip conditions apply [1]. At steady state operations the time derivative goes to zero and is achieved when the pressure wave having half of the sliding velocity travels across the length of the bearing [15].

The extreme values of the clearance (i.e., $\sim 10^{-8} \mathrm{~m}$ ) require a correction to the conventional flow theory, which assumes the flow velocity at the boundary to equal the boundary velocity. This "no-slip" or continuum theory applied to compressible fluid at ultra-low clearances, the continuum Reynolds equation fails to deliver reasonable solutions. For Knudsen number $K n<<1$, slip flow accurately models compressible gas lubrication conditions. Transitional flow occurs for $K n$ between 0.1 and 10; for even larger $K n$, free molecular flow conditions exist in the bearings. The correction is made through the flow factor, 
Q. Applying the above assumptions and conditions, Navier-Stokes and continuity equations with appropriate correction factors yield [12]:

$$
\nabla \cdot\left(Q h^{3} P \nabla P\right)=6 \mu V \cdot \nabla P h+12 \mu \frac{\partial P h}{\partial t}
$$

where $h, P, \mu, V, t$, and $Q$ represent the characteristic length of the flow, bearing pressure, viscosity, velocity, time, and flow factor, respectively. With $Q=1$, the equation reduces to a continuum model. For this study, we use the first order slip model $Q=1+\left(6 a K n_{0}\right) /(P H)$, where $a=(2-\alpha) / \alpha$ is the accommodation factor.

Although the finite element method (FEM) has been used in the solution of the Reynolds equation [14], the finite difference method appears to be the method of choice for many researchers $[3,4,6,9]$. Singular perturbation techniques for asymptotic solution [5] had also been tried but had not gained popular support. In addition to the above numerical techniques, a widely known alternating direction implicit method is chosen to solve the Reynolds equation with first-order slip in this study. A factored implicit scheme (FIS) outlined in White and Nigam [15] is used with corrections made to the first del operator.

$$
\nabla \cdot(h P \nabla P)+6 \lambda_{a} P_{a} \nabla \cdot\left(h^{2} \nabla P\right)=6 \mu V \cdot \nabla P h+12 \mu \frac{\partial P h}{\partial t}
$$

where $h, P, \lambda_{a}, P_{a}, \mu$, and $t$ represent the gas bearing spacing, gas bearing pressure, mean free path of the gas at ambient pressure, ambient pressure, lubricant viscosity, and time, respectively.

The bearing number, $\Lambda$, is a nondimensional quantity measuring the ratio of Couette flow to Poiseulle flow. Continuum solutions are invalid for high $\Lambda$. This question has been addressed through molecular gas film lubrication [6]. In traditional finite difference schemes with uniform meshes nonphysical oscillations propagate throughout the fluid, rendering the numerical solution useless. The question is addressed through variable meshing [15].

\section{Lift effects on particle motion}

A slider of length, $l$, and pitch, $\theta$, rides above a disk spinning at $\hat{\Omega}$ (or slides with a linear velocity $\hat{U}$ ) is presented in fig. 1 . The slider and disk are separated by $h$. The minimum clearance $\left(h_{m}\right)$, slider length, and pitch angle are taken in the neighborhood of $3 \mu \mathrm{m}, 2 \mathrm{~mm}$, and $150 \mu \mathrm{rad}$, respectively. A spherical of diameter (100 to $350 \mathrm{~nm}$ ), $d$, enters the air bearing.

The motion of a particle inside an air bearing can be described fully by its position vector $\dot{x}_{p}\left(x_{p}, y_{p}, z_{p}\right)$ and velocity vector $\dot{v}_{p}\left(u_{p}, v_{p}, w_{p}\right)$. Time derivatives result in six coupled differential equations that are solved simultaneously at each time step.

Zhang and Bogy $[17,18]$ considered the effects of lift on the motion of particles in the recessed region of a slider. This study examined four important forces inside the air bearing - drag, Saffman, Magnus, and gravity forces. Their 


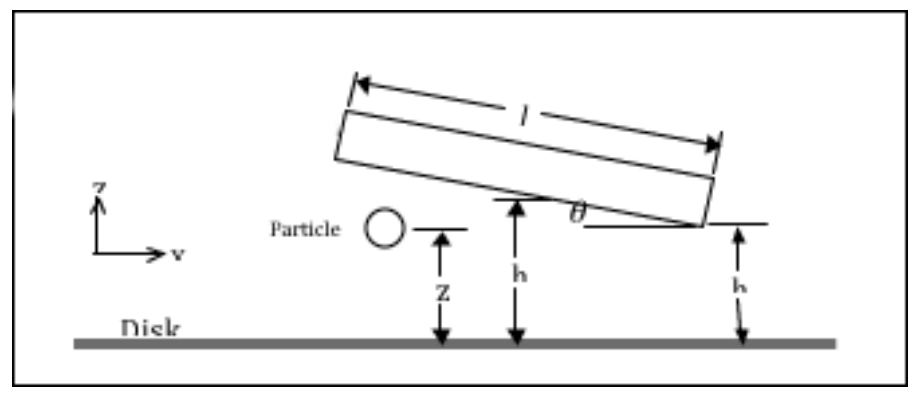

Figure 1: $\quad$ Simplified slider-disk assembly.

investigation revealed a relationship between the lift forces and particle size, relative velocity, and particle density.

Liu and his colleagues [11] used the Boltzmann equation to study the kinetic theory of sphere drag in transition flows. In addition to particle drag, the particle can be "lifted" in the direction perpendicular to the fluid flow, known as the Saffman lift force. If particle velocity is greater than fluid velocity, the force will point upward toward the slider, and vice versa [13]. This result is valid for very small Reynolds numbers. As the sphere flows through air, the gravitational pull (or push) is of order $d^{3}$. Compared to the drag components in the $x-y$ plane, which is of order $d^{2}$, only the $z$-component is significant.

The fourth lift force considered by Zhang and Bogy is the Magnus force stemming from a spin of the sphere in a fluid. Mangus force, however, is an order of magnitude smaller than Saffman force and is not considered in the present study.

\section{Effects of electrostatic charges on particle motion}

It is difficult to measure exactly how much electrostatic charge is present in a contaminant particle. Consequently, the Bohr radius is used to estimate the maximum charge on an aluminum sphere. Given the diametrical range of 100$350 \mathrm{~nm}$, a $100 \mathrm{~nm}$ aluminum sphere may hold roughly 844 million hydrogen atoms or equivalents. A 350nm sphere can hold in excess of 36 billion hydrogen atoms or equivalents. Assuming that each hydrogen atom is ionized, 100-350nm aluminum sphere can be charged on the order of $1.3516 \times 10^{-10}$ Coulombs (C) and $5.7950 \times 10^{-9} \mathrm{C}$, respectively. Coulomb's Law gives the electrostatic force:

$$
f_{E}=\frac{1}{4 \pi \varepsilon_{0}} \frac{Q_{p} Q^{*}}{r^{2}}=8.9918 \times 10^{6} \frac{Q_{p} Q^{*}}{r^{2}}
$$

where $Q_{p}$ and $Q^{*}$ measure electrostatic charges on the particle and slider (or disk). The distance between $Q_{p}$ and $Q^{*}$ is the value, $r$.

Drag, Saffman, gravity, and electrostatic forces combine to influence particle motion. Saffman, gravity, and electrostatic forces all act in the direction perpendicular to the fluid flow. 


$$
\dot{F}_{p}=f_{L(\text { drag,Saffman, }, \text { ravity,Magnus })}+f_{E}
$$

Dimensional coordinates and bearing parameters are made dimensionless. Horizontal components are normalized with respect to slider length, and vertical components are divided by minimum height. The product of rotational speed $\hat{\Omega}$ and circumference yields the linear sliding velocity $\hat{U}$. Velocity components are normalized by $\hat{U}$, and time is multiplied by $\hat{\Omega}$ to produce T:

$$
X=\frac{x}{l} \quad U=\frac{u}{\hat{U}} \quad T=\hat{\Omega} t
$$

Applying the chain rule conveniently transforms other dimensional variables into dimensionless variables. For example,

$$
\frac{d X_{p}}{d T}=\frac{d x_{p}}{d t} \frac{d t}{d T} \frac{d X_{p}}{d x_{p}}=\frac{\hat{U}}{\hat{\Omega} l} U_{p}, \quad \frac{d Y_{p}}{d T}=\frac{\hat{U}}{\hat{\Omega} l} V_{p}, \quad \frac{d Z_{p}}{d T}=\frac{\hat{U}}{\hat{\Omega} l} W_{p}
$$

Similarly, the acceleration components in the three orthogonal coordinates are also given by the chain rule. The $Z$-component acceleration has contributions from drag, Saffman, gravity, and electrostatic forces [12]:

$$
\begin{aligned}
\frac{d W_{p}}{d T}= & \frac{3}{4} \frac{\hat{U} h_{m}}{\rho_{g}} \frac{\rho_{D}}{D} \bar{U}\left(W_{g}-W_{p}\right)+\frac{9.69}{\pi} \frac{\hat{U} \tilde{U}}{\hat{\Omega} h_{m} D} \frac{\rho_{g}}{\rho_{p}} \sqrt{\frac{v_{g} h_{m} \kappa}{\hat{U}^{2} h_{m}}} \\
& +\frac{\hat{U}}{\hat{\Omega} h_{m}}\left(\frac{\rho_{g}}{\rho_{p}}-1\right) \frac{h_{m}}{\hat{U}^{2}} g+\frac{1.717308 \times 10^{10}}{\rho_{p} r^{2} h_{m}{ }^{3} D^{3}} \frac{Q_{p} Q^{*}}{\hat{\Omega} \hat{U}}
\end{aligned}
$$

where $C_{D}, \rho_{g}, \kappa, v_{g}, \bar{U}=\frac{\left|\bar{v}_{g}-\bar{v}_{p}\right|}{\hat{U}}, D=\frac{d}{h_{m}}$, and $\tilde{U}=\frac{\Delta U}{\hat{U}}$ represent drag coefficient, gas density, velocity gradient, gas velocity, quotient of the velocity norm and sliding velocity, and dimensionless particle diameter, and dimensionless velocity, respectively.

A system of six coupled differential equations at each time step, $\Delta T$, are solved using the classical Runge-Kutta method. Once solutions at time step $n+1$ are found, the time derivatives in eqn. 6 are rewritten as simple slopes:

$$
\frac{d X_{p}}{d T} \cong \frac{X_{p(n+1)}-X_{p(n)}}{\Delta T}, \frac{d Y_{p}}{d T} \cong \frac{Y_{p(n+1)}-Y_{p(n)}}{\Delta T}, \frac{d Z_{p}}{d T} \cong \frac{Z_{p(n+1)}-Z_{p(n)}}{\Delta T}
$$

The slope equations above accurately approach the true value of the derivatives using a very small time step, $\Delta T$. Once positions at time step $(n+1)$ are solved, all parameters in the acceleration equations are updated then iterated. The 
iterations stop once the particle has either passed out of the air bearing or has impacted on the surfaces.

\section{Numerical results}

The air bearing used in this study has a typical cavity depth of $3 \mu \mathrm{m}$ and a $50 \mathrm{~nm}$ flying height. Disk speed in the $x$-direction, $\hat{U}$, was set at $20 \mathrm{~m} / \mathrm{s}$. The particles chosen were assumed to consist of aluminum spheres with a density, $\rho_{\mathrm{p}}$, of $4000 \mathrm{~kg} / \mathrm{m}^{3}$. Diameters of the particles used began at $150 \mathrm{~nm}$, which was incremented by $50 \mathrm{~nm}$ for each new simulation, to a maximum particle diameter of $300 \mathrm{~nm}$. The following parameters were used for each simulation: initial position, $X_{p}=0, Y_{p}=$ constant, and $Z_{p}=0.5$; initial velocity in $Y$-direction, $V_{p}=0$; initial velocity in $Z$-direction, $W_{p}=0$.

Initial particle velocity, $U_{p}$ remains between 0.25 and 1.0 at the entrance to the bearing. Initial particle speeds slower than 0.25 or faster than 1.0 (1.0 is equal to the speed of the disk) are unlikely to occur in real world environments; therefore, although transport solutions are attainable for these numbers, they are trivial.

The results of the initial runs for trace number 1 (no electrostatic input) in figs. 2-5 agree with findings by Zhang and Bogy in their previous work because the Saffman lift force increases according to the square of the particle radius. Thus, particles below $100 \mathrm{~nm}$ are negligibly affected. Electrostatic force was added in successive runs until the particle first impacted with either the slider or the disk. One equivalent $\mathrm{H}+$ ion contains the same amount of charge as one electron, or $1.602 \times 10^{-19} \mathrm{C}$. The measurement of charge in equivalent $\mathrm{H}+$ ions is merely a convenient method to change the electrostatic charge of the particle within the confines of a numeric program. The use of equivalent $\mathrm{H}+$ ions also puts an upper bound on the amount of maximum charge the particle can acquire. Through the use of the Bohr's radius of $5.29167 \times 10^{-11} \mathrm{~m}$, the volume of a hydrogen atom, can be calculated. The volume of a particle sphere is divided by

Table 1: Results from particle motion study.

\begin{tabular}{|c|c|c|c|}
\hline Diameter $(\mathrm{nm})$ & Trace No. & H+ Ions & Charge $\left(\times 10^{18 \mathrm{C})}\right.$ \\
\hline 150 & 2 & 11.4 & 1.82 \\
\hline 150 & 3 & 19.0 & 3.04 \\
\hline 150 & 4 & 35.6 & 5.70 \\
\hline 200 & 2 & 9.6 & 1.54 \\
\hline 200 & 3 & 16.9 & 2.70 \\
\hline 200 & 4 & 33.7 & 5.41 \\
\hline 250 & 2 & 1.5 & 2.35 \\
\hline 250 & 3 & 4.4 & 7.04 \\
\hline 250 & 4 & 13.2 & 2.11 \\
\hline 300 & 2 & 2.3 & 3.65 \\
\hline 300 & 3 & 20.7 & 3.32 \\
\hline 300 & 4 & 28.5 & 4.56 \\
\hline
\end{tabular}


the volume of an $\mathrm{H}+$ ion to determine the maximum number of ions possible in a given diameter.

Fig. 2 is a plot of the motion study results for a particle diameter of $150 \mathrm{~nm}$. The first impact occurred at an equivalent $\mathrm{H}+$ ion number of 11.4. This is only a fraction of the maximum possible number of $\mathrm{H}+$ ions of $2.85 \times 10^{9}$. Trace (3) impacted at $\mathrm{H}+$ ions equal to 19.0 and trace (4) impacted at $\mathrm{H}+$ ions equal to 35.6. Note the electrostatic force in trace number 4 exceeded the small Saffman force and impacted the disk.

The results for a particle diameter of $200 \mathrm{~nm}$ are represented in fig. 3. Initial particle speed for all runs of $d=200 \mathrm{~nm}$ was 1.0. The first impact occurred at an equivalent $\mathrm{H}+$ ion count of 9.6. Successive impacts were noted at $\mathrm{H}+$ counts of 16.8 and 33.7 .

Fig. 4 shows results from the study of a particle with a diameter of $250 \mathrm{~nm}$ for initial speed of 1.0. The effect of the Saffman force dominating drag and gravity is shown through the sharp upward swing of trace (1). The number of equivalent $\mathrm{H}+$ ions to first impact on trace (2) is only 1.5. This is a significant decrease. Trace (4) impact occurred at 13.2 equivalent $\mathrm{H}+$ ions.

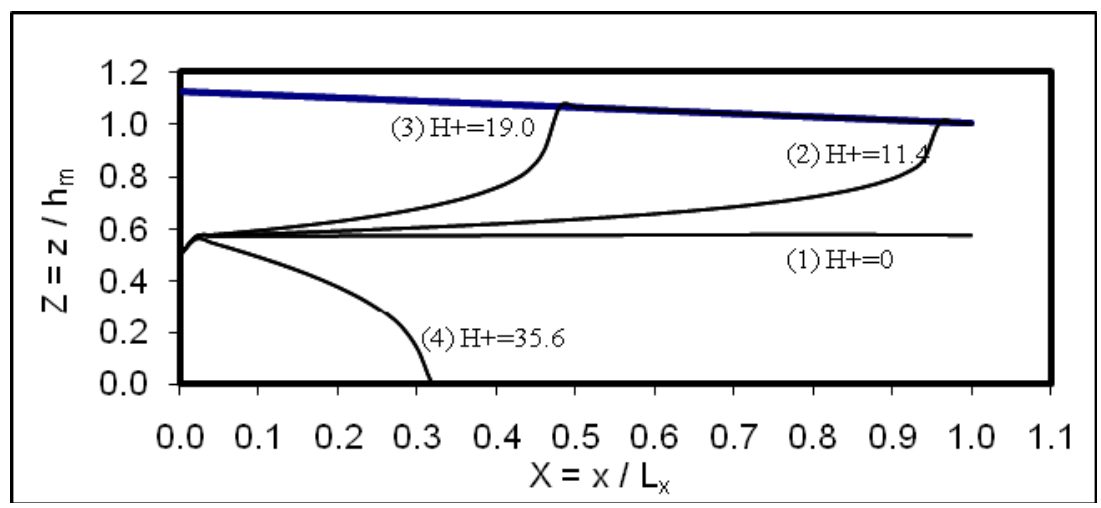

Figure 2: $\quad$ Particle diameter 150nm.

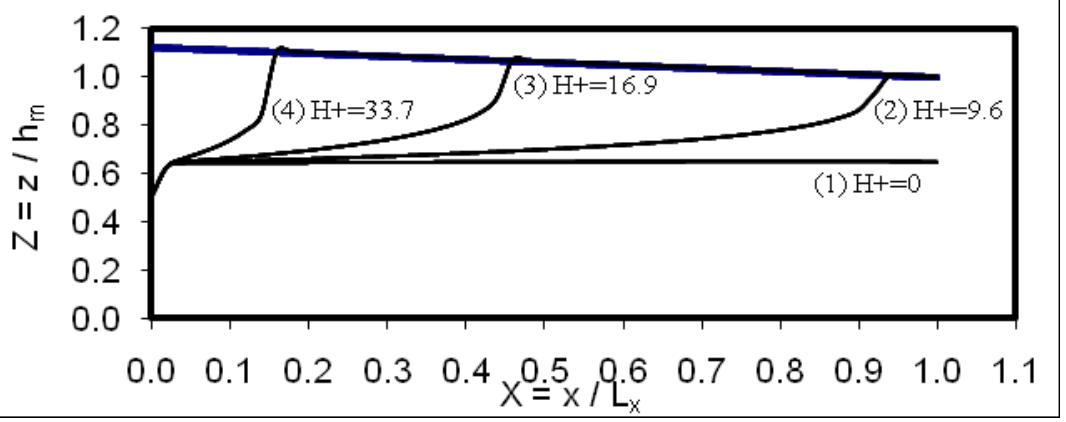

Figure 3: $\quad$ Particle diameter 200nm. 
The last particle size studied has a diameter of $300 \mathrm{~nm}$. The results are plotted as fig. 5. At this particle size and larger, gravity and drag forces are insignificant. At this particle size the trajectory is very sensitive to the initial speed. However, the first impact occurred at an equivalent $\mathrm{H}+$ ion count of 2.3, which is slightly higher than the first impact of the particle of fig. 4. This may be due to discrepancies in the model resulting from the differing initial speed, but is more likely to be caused by the particle's increasing mass. Successive impacts occurred at $\mathrm{H}+$ counts of 20.7 and 28.5. These counts too are higher than the $d=250 \mathrm{~nm}$ particle, but note that the Saffman force has been exceeded with impact occurring on the disk.

\section{Conclusions}

If electrostatic charges are present in the air bearing, they can be a significant factor in the path taken by a particle. All particle trajectories through the air bearing were affected at lower charges than anticipated, which makes the

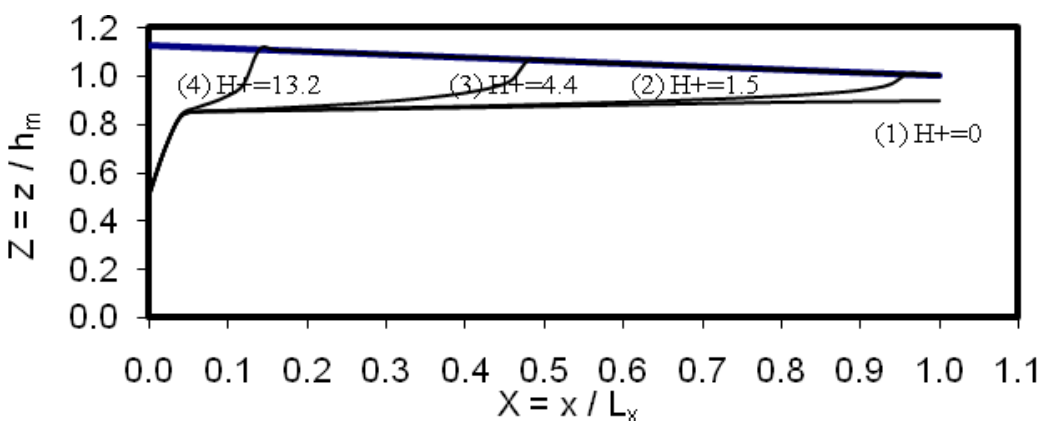

Figure 4: $\quad$ Particle diameter 250nm.

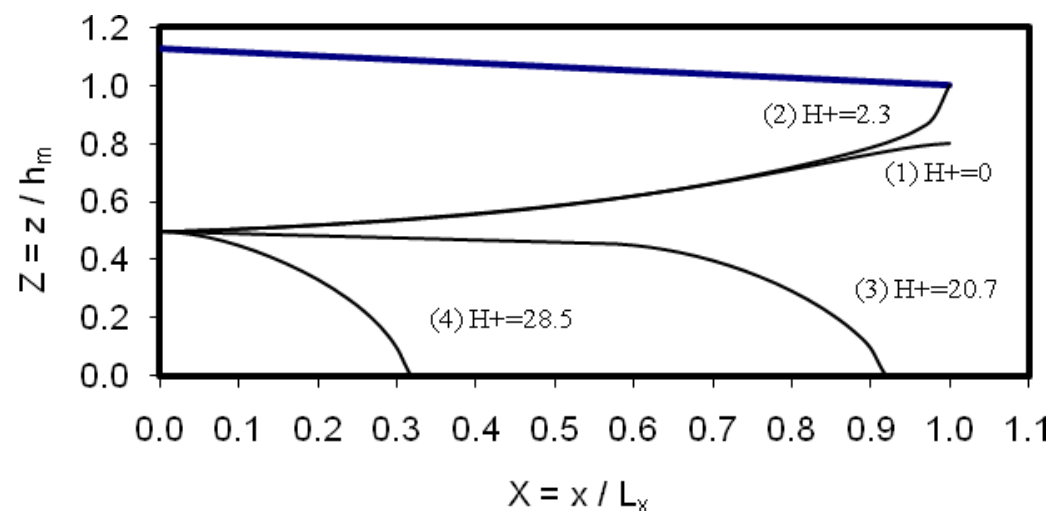

Figure 5: $\quad$ Particle diameter 300nm. 
findings of this study very significant. Only a minute charge is needed to induce a trajectory change. This small magnitude $\left(\sim 1.5 \mathrm{H}+\right.$ ions, or $\left.2.35 \mathrm{x} 10^{-19} \mathrm{C}\right) \mathrm{might}$ be sufficient to ionize particles that exert an electrostatic force. Electrostatic charges are very likely present at these small magnitudes. High-speed debris flows are well known to produce electrostatic charges.

If electrostatic forces are generated within the bearing, then there are surely instances when even fewer charges than simulated can be present. Such an amount will affect a trajectory change. The initial particle height in all trial runs was set at a conservative $Z_{p}=0.5$. Trial runs conducted at initial height closer to the disk or slider have resulted in trajectory changes at some very low magnitudes.

It is proposed that electrostatic forces could explain why debris tends to accumulate in the cavity area. If such debris enters the cavity area on the slider, a charged particle would tend to remain.

Understanding particle path changes at very low electrical charges could be significant. In the presence of electrostatic charges in modern air bearings, contaminant control methods in the hard disk drive environment will need to be devised. What effects do oscillating charges have on particle motion? To illustrate, after writing the particle transport program, the first initial runs produced a few particle paths that oscillated between the disk and plate surfaces like a sine wave. Realizing such an event is a low probability it was discovered the routine that examined the distance $r$ in the electrostatic force was reversed to select the longest dimension. This reversed the direction of the electrostatic force and produced repulsion. If the slider could push the particles, impact damage on the disk surface and debris accumulation on the slider could be possible.

\section{Dedication and acknowledgement}

This paper is dedicated to Dr. G. Steven Gipson who passed away on April 22, 2009 after a long courageous battle with pancreatic cancer. Over the past three decades he made significant contributions in engineering mechanics at all scales, especially in boundary element methods. The authors acknowledge Seagate Technology for initial funding for this work.

\section{References}

[1] Bhushan, B., (ed.), Tribology and Mechanics of Magnetic Storage Devices, New York, Springer-Verlag, 1990.

[2] Cameron, A., Basic Lubrication Theory, 3rd ed., Wiley: New York, Wiley, 1981.

[3] Castelli, V. \& Pirvics, J., Review of Numerical Methods in Gas Bearing Film Analysis. Journal of Lubrication Technology, pp. 778-792, 1968.

[4] Coleman, R, The Numerical Solution of Linear Elliptic Equations. Journal of Lubrication Technology, pp. 773-776, 1968. 
[5] DiPrima, R.C., Asymptotic Method for an Infinitely Long Slider SqueezeFilm Bearing. Journal of Lubrication Technology, pp. 173-183, 1968.

[6] Fukui, S. \& Kaneko, R., Analysis of Ultra-Thin Gas Film Lubrication Based on Linearized Boltzmann Equation: First Report-Derivation of a Generalized Lubrication Equation Including Thermal Creep Flow. Journal of Tribology, 110, pp. 253-262, 1988.

[7] Gross, W., Matsch L., Castelli, V., Eshel, A., Vohr, J. \& Wildmann, M., Fluid Film Lubrication, Wiley: New York, 1980.

[8] Hiller B. \& Singh, G., Interaction of Contamination Particles With the Particulate Slider/Disk Interface. Advanced Information Storage Systems, 173(2), 1991.

[9] Hu, Y. \& Bogy, D., Dynamic Stability and Spacing Modulation of Sub25nm Fly Height Sliders. Journal of Tribology, 119, pp. 646-652, 1997.

[10] Koka, R. \& Kumaran, A., Visualization and Analysis of Particulate Buildup on the Leading Edge Taper Sliders. Advanced Information Storage Systems, 161(2), 1991.

[11] Liu, V., Pang, S. \& Jew, H., Sphere Drag in Flows of Almost-Free Molecules. Physics of Fluids, 8(5), pp. 788-796, 1965.

[12] Polwort, R., Motion of Nanoscale Contaminant Particles in Air Bearings, Master's thesis, Oklahoma State University, 1999.

[13] Saffman, P., The Life on a Small Sphere in a Slow Shear Flow. Journal of Fluid Mechanics, 22(2), pp. 385-400, 1965.

[14] Tokuyama, M. \& Shinichi, H., Dynamic Flying Characteristics of Magnetic Head Slider With Dust. Journal of Tribology, 116, pp. 95-100, 1994.

[15] White, J. \& Nigam, A., A Factored Implicit Scheme for the Numerical Solution of the Reynolds Equation at Very Low Spacing. Transactions of the ASME, 102, pp. 80-85, 1980.

[16] Williams, J., Engineering Tribology, Oxford: New York, 1994.

[17] Zhang, S. \& Bogy, D., Effects of Lift on the Motion of Particles in the Recessed Regions of a Slider. Physics of Fluids, 9, pp. 1265-1272, 1997.

[18] Zhang, S. \& Bogy, D., Slider Designs for Controlling Contamination. Journal of Tribology, 119, pp. 537-540, 1997. 